\title{
Research Article \\ Effects of Parasitic Fractional Elements to the Dynamics of Memristor
}

\author{
Rawid Banchuin \\ Faculty of Engineering and Graduated School of Information Technology, Siam University, Bangkok, Thailand \\ Correspondence should be addressed to Rawid Banchuin; rawid_b@yahoo.com
}

Received 25 March 2019; Revised 27 May 2019; Accepted 4 June 2019; Published 27 June 2019

Academic Editor: Muhammad Taher Abuelma'atti

Copyright (C) 2019 Rawid Banchuin. This is an open access article distributed under the Creative Commons Attribution License, which permits unrestricted use, distribution, and reproduction in any medium, provided the original work is properly cited.

In this research, we study the effects of the parasitic fractional elements to the dynamic of the memristor where both flux- and charge-controlled memristors have been considered. For doing so, the fractional differential equation-based approach has been used for modeling the memristor and the memristor-based circuits under the effects of the parasitic fractional elements where the resulting equations have been solved both analytically and numerically. From the obtained solutions and simulations, the effects of the parasitic fractional elements to the dynamic of the memristor have been studied. We have found that the parasitic fractional elements cause the charge and flux decay of the memristor similarly to the conventional parasitic elements. Moreover, the impasse points of the phase portraits between flux and charge of the memristor-based circuits can also be broken by the parasitic fractional elements. The effects of the order and the nonlinearity of the parasitic fractional elements have also been studied.

\section{Introduction}

Memristor is the 4th electrical circuit element that has been theoretically found by Leon Chua since 1971 [1]. The memristor can be simply thought of as the resistor with memory. For decades after Chua proposed his original work, the memristor has been practically realized by a research group in Hewlett Packard (HP) labs [2] in 2008. The memristor has been applied in various applications, e.g., parametric oscillator, DRAM, signal processing, neural networks, programmable logic, cross bar switch, passive switch, and control systems. [3-6]. According to the importance of the memristor, the studies in its circuit theoretical aspects have been proposed [7-10]. In [9], the effects of the parasitic element to the memristor have been studied where it has been found that such parasitic element exhibits significant effects to the memristor and memristor-based circuits. However, it was assumed that the orders of the parasitic elements are strictly integers despite that they can be fractional in practice as those parasitic element can also be the fractional elements or fractances [11-13].

Therefore, we study the dynamic of the memristor and the memristor-based circuits under the effects of parasitic elements, which are the fractional elements, in this work where comparison with [9] has also been made. For simplicity, we appeal the parasitic elements, which are the fractional elements, as the parasitic fractional elements. Here, both flux- and charge-controlled memristors have been considered. Since the order of the parasitic fractional element can be fractional, the fractional differential equation (FDE), which its order can be fractional, must be used for modeling the memristor and the memristor-based circuits under the effects of such parasitic fractional element instead of the ordinary differential equation (ODE) which its order is strictly integer. The FDE is the extension of the ODE in the domain of fractional calculus which plays a fundamental role in various engineering fields, e.g., bioengineering, control theory, electronics, robotics, and signal processing [14-18]. The analytical solutions of the formulated FDEs, which are nonlinear, have been determined by using the Adomian decomposition method [19]. On the other hand, the numerical solutions have been obtained by using the integer derivative-based approximation of the fractional derivative with the moments of a function [20]. From the obtained solutions and simulations with MATHEMATICA, the effects of the parasitic fractional element to the dynamic of the memristor and the memristor-based circuits have been studied. We have found that the parasitic fractional element 
also causes the charge and flux decay of the memristor and breaks the impasse points of the phase portraits between flux and charge of the memristor-based circuits. Moreover, the effects of the order and the nonlinearity of the parasitic fractional element have also been studied.

In the following section, some background theory on the memristor and the fractional element will be briefly given. The flux and charge decaying of the memristor due to the parasitic fractional element will be illustrated in Section 3 followed by the impasse points breaking of the phase portraits between flux and charge of the memristor-based circuits in Section 4 where the effects of the order of the parasitic fractional element will be studied in these sections. The nonlinear effect of the parasitic fractional element will be explored in Section 5, and the conclusion will be finally drawn in Section 6.

\section{Background Theory}

2.1. Memristor. Memristor is a nonlinear electrical circuit element. This circuit element relates the flux $(\varphi)$ and charge (q) through one of the following constitutive relations [9]:

$$
\begin{aligned}
& \varphi=f(q), \\
& q=g(\varphi) .
\end{aligned}
$$

Noted that both $f(\cdot)$ and $g(\cdot)$ must be differentiable and scalar-valued. Moreover, the memristor with its constitutive relation given by (1a) and (1b) can be referred to as the $q$ controlled memristor and the $\varphi$-controlled memristor, respectively. According to [21], the ideal memristor is nonvolatile. Therefore, it can retain the values of $\varphi$ and $q$ at the moment that it is switched off, i.e., $\varphi$ and $q$ are always conserved without any change as time goes by. This property of the ideal memristor corresponds to the fundamental circuit law on $\varphi$ and $q$, namely, the principle of the conservation of charge and flux [22].

From (1a) and (1b), the following mathematical expression of small-signal memristance, $M(q)$, and memductance, $W(\varphi)$, of the memristor can be obtained [9]:

$$
\begin{aligned}
& M(q)=\frac{d f(q)}{d q}, \\
& W(\varphi)=\frac{d g(\varphi)}{d \varphi} .
\end{aligned}
$$

2.2. Fractional Element. Fractional element is a circuit element with the impedance function of fractional order, $\alpha$, where $0<\alpha<1$. According to [23], the fractional element which the phase of its voltage always leads that of its current is referred to as the fractional inductor. On the other hand, such element which the phase of its voltage always lags that of its current is referred to as fractional capacitor. For the fractional inductor, its s-domain impedance function can be given by

$$
Z_{L_{\alpha}}=L_{\alpha} s^{\alpha}
$$

where $L_{\alpha}$ denotes the inductivity of the fractional inductor [24]. On the other hand, we have the following impedance function for the fractional capacitor:

$$
Z_{C_{\alpha}}=\left(C_{\alpha} s^{\alpha}\right)^{-1}
$$

where $C_{\alpha}$ stands for the pseudocapacitance of the fractional capacitor [25].

By using (4) and (5), the following flux-charge relationships can be, respectively, obtained for the fractional inductor and fractional capacitor:

$$
\begin{aligned}
& \varphi_{L_{\alpha}}=L_{\alpha} D^{\alpha}\left[q_{L_{\alpha}}\right], \\
& q_{C_{\alpha}}=C_{\alpha} D^{\alpha}\left[\varphi_{C_{\alpha}}\right],
\end{aligned}
$$

where $D^{\alpha}[]$ denotes the fractional derivative operator. Moreover, $\varphi_{C_{\alpha}}\left(\varphi_{L_{\alpha}}\right)$ and $q_{C_{\alpha}}\left(q_{L_{\alpha}}\right)$ stand for the flux and charge of the fractional capacitor (fractional inductor).

\section{Flux and Charge Decay by the Parasitic Fractional Elements}

Despite the conservation of $\varphi$ and $q$ in the ideal phenomena, the memristor with parasitic elements exhibits the decaying of both $\varphi$ and $q$ after being switched off as proposed in [9]. In this work, we will show that such decaying of both $\varphi$ and $q$ can also be occurred by the parasitic fractional elements.

Firstly, the parasitic fractional capacitor will be considered. The memristor with parasitic fractional capacitor can be modeled as an ideal memristor connected in parallel with a fractional capacitor as depicted in Figure 1; thus, we have $\varphi_{C_{\alpha}}=\varphi$. If we assume that the memristor is $\varphi$-controlled, the following relationship can be obtained by using the conventional circuit analysis after the memristor is switched off:

$$
q_{C_{\alpha}}=-g(\varphi) .
$$

By using (1b), (7), and (8), the following FDE can be obtained:

$$
D^{\alpha}[\varphi]=-\frac{q}{C_{\alpha}} .
$$

Therefore, we have found that

$$
q \propto-D^{\alpha}[\varphi]
$$

On the other hand, the relationships of the memristor affected by parasitic conventional capacitor, $C$, have been proposed in [9] as follows:

$$
\begin{aligned}
& \frac{d \phi}{d t}=-\frac{q}{C}, \\
& q \propto-\frac{d \phi}{d t} .
\end{aligned}
$$

Since the time domain fractional derivative is also referred to as the rate of change similarly to ordinary derivative, it can be found from (10) that $\varphi$ of the memristor affected by the parasitic fractional capacitor is decreased 


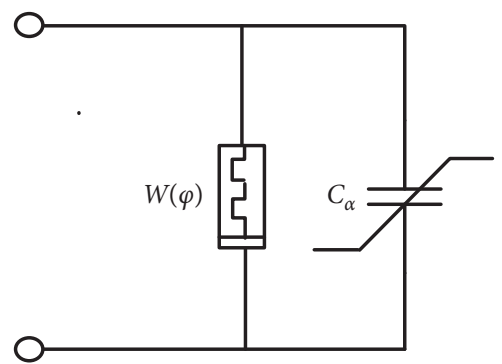

FIgURE 1: Equivalent circuit model of the memristor affected by the parasitic fractional capacitor.

with respect to time when $q>0$ and vice versa similarly to that of the parasitic conventional capacitor-affected device as proposed in [9] and can be seen in this work from (11). Here, we assume that $q=g(\varphi)$ lies in the first and third quadrants of $\varphi-q$ plane as it is a strictly increasing function and $g(0)=$ 0 as also assumed in [9]. After applying the dynamic route theory [26] to (10), we have found that

$$
\lim _{t \rightarrow \infty}[\varphi]=0 \text {. }
$$

Thus, it can be stated that the parasitic fractional capacitor causes the decaying of $\varphi$ after the memristor is switched off similarly to the parasitic conventional capacitor as proposed by [9]. Since $q=g(\varphi)$ is a strictly increasing function, we have

$$
\lim _{t \rightarrow \infty}[q]=0
$$

which states the decaying of $q$ is occurred by the parasitic fractional capacitor as well as by the parasitic conventional capacitor [9]. It should be mentioned here that both (13) and (14) can also be obtained by applying the dynamic route theory to (11) and keeping in mind that $q=g(\varphi)$.

Now, consider the parasitic fractional inductor. The memristor with parasitic fractional inductor can be modeled as an ideal memristor connected in series with a fractional inductor as depicted in Figure 2; therefore, $q_{L_{\alpha}}=q$. By assuming that the memristor is $q$-controlled and applying (1a) and (6), the following equation can be obtained after the memristor is switched off:

$$
D^{\alpha}[q]=-\frac{\varphi}{L_{\alpha}} .
$$

From (15), it can be seen that

$$
\varphi \propto-D^{\alpha}[q]
$$

which shows that $q$ is decreased with respect to time when $\varphi>0$ and vice versa. If we assume that $\varphi=f(q)$ lies in the first and third quadrants of $q-\varphi$ plane and $f(0)=0$, we have found that

$$
\lim _{t \rightarrow \infty}[q]=0
$$

by using the dynamic route theory.

As $\varphi=f(q)$ is a strictly increasing function, we also have

$$
\lim _{t \rightarrow \infty}[\varphi]=0 \text {. }
$$

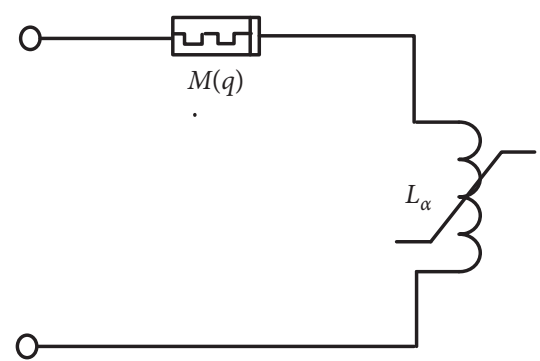

FIGURE 2: Equivalent circuit model of the memristor affected by the parasitic fractional inductor.

Therefore, it can be seen that the parasitic fractional inductor induces the decaying of $q$ which in turn causes the decaying of $\varphi$ when the memristor is switched off similarly to the parasitic conventional inductor as proposed in [9] by using the intuition without the mathematical proof.

As a practical illustration, let the constitutive relation of the memristor be specifically given by $q=\varphi^{3}$. Therefore, the effect of the parasitic fractional capacitor can be analytically described by the following nonlinear FDE:

$$
D^{\alpha}[\varphi]=\frac{\varphi^{3}}{C_{\alpha}},
$$

which can be derived by using (9) and the above constitutive relation.

If the parasitic conventional capacitor has been considered on the other hand similarly to the previous study [9], its effect can be described by the following ODE:

$$
\frac{d \varphi}{d t}=\frac{\varphi^{3}}{C}
$$

By using the Adomian decomposition, the following analytical solution of (17) can be obtained:

$$
\varphi=\varphi_{0}+\varphi_{1}+\varphi_{2}+\varphi_{3} \cdots,
$$

where $\varphi_{0}$ denotes the initial value of $\varphi($ at $t=0)$. On the other hand, the higher order terms can be given in terms of $\varphi_{0}$ as follows:

$$
\begin{aligned}
& \varphi_{1}=-\frac{\varphi_{0}^{3} t^{\alpha}}{C_{\alpha} \Gamma(\alpha+1)} \\
& \varphi_{2}=\frac{3 \varphi_{0}^{5} t^{2 \alpha}}{C_{\alpha}^{2} \Gamma(2 \alpha+1)}, \\
& \varphi_{3}=-\frac{\varphi_{0}^{7} t^{3 \alpha}\left(3(\Gamma(\alpha+1))^{2}+\Gamma(2 \alpha+1)\right)}{C_{\alpha}^{3} \alpha(\Gamma(\alpha+1))^{2} \Gamma(3 \alpha)},
\end{aligned}
$$

where $\Gamma(\cdot)$ stands for the gamma function [26].

For determining the numerical solution of (20), the integer derivative-based approximation of $D^{\alpha}[\varphi]$ by using the moments of a function has been used. As a result, the following system of ODE can be obtained: 


$$
\begin{aligned}
\frac{d \varphi}{d t} & =\frac{\left(-\varphi^{3} / C_{\alpha}\right)+\sum_{p=2}^{N}\left[\left(\Gamma(p-1+\alpha) t^{1-p-\alpha} V_{p}\right) /(\Gamma(2-\alpha) \Gamma(\alpha-1)(p-1) !)\right]-\left(\left(1+\sum_{p=2}^{N}[(\Gamma(p-1+\alpha)) /(\Gamma(\alpha)(p-1) !)]\right) /(\Gamma(1-\alpha))\right) t^{-\alpha} \varphi}{\left(1+\sum_{p=1}^{N}[((\Gamma(p-1+\alpha)) / \Gamma(\alpha-1) p !)]\right) /(\Gamma(2-\alpha)) t^{1-\alpha}} \\
\frac{d V_{2}}{d t} & =-\varphi \\
\frac{d V_{3}}{d t} & =-2 t \varphi \\
\quad & \\
\frac{d V_{N}}{d t} & =(1-N) t^{N-2} \varphi
\end{aligned}
$$

where the initial values of $V_{2}, V_{3}, \ldots, V_{N}$ have been automatically assumed to be 0 due to the adopted approximation methodology [21].

Here, we let $N=7$ in (23) as this value of $N$ gives a satisfied approximation of the fractional derivative [20] while being not too large; thus, the numerical solution of (17), i.e., $\varphi$, can be obtained by also maintaining a reasonable amount of computational effort. The obtained $\varphi$ can be simulated against $t$ for various values of $\alpha$ as depicted in Figure 3 where $\varphi_{0}=1$ and $C_{\alpha}=0.01$ have been assumed. Similarly to [7-10], all related quantities have been given in dimensionless manner without loss of generality as this work also focuses on the circuit theoretical aspect of memristor. Since $q=\varphi^{3}, q$ can be simulated as depicted in Figure 4 where the similar assumption has been adopted. From these figures which $\varphi$ and $q$ of the memristor affected by the conventional parasitic element [9] which can be simulated here by using the numerical solution of (20) have also been included for comparison, the decaying of both $\varphi$ and $q$ due to the parasitic fractional element can be clearly observed. This confirms the aforementioned dynamic route theory based on generic results. Moreover, we have also found from Figures 3 and 4 that the decaying rates of $\varphi$ and $q$, which are much faster than those of the $\varphi$ and $q$ decaying caused by the conventional parasitic element, are inversely proportional to $\alpha$. This is because the discharging rate of the fractional capacitor and the flux decaying rate of the fractional inductor which serve as the parasitic elements in this scenario are inversely proportional to $\alpha$. For a demonstration, consider a resistor connected in parallel with the fractional capacitor as shown in Figure 5. After the resistor being switched off, the following linear FDE can be obtained by applying the conventional circuit analysis where $R$ stands for the resistance of the resistor:

$$
D^{\alpha}\left[q_{C_{\alpha}}\right]+\frac{q_{C_{\alpha}}}{R C_{\alpha}}=0
$$

After the Laplace transformation and inverse Laplace transformation, we have

$$
q_{C_{\alpha}}=q_{0 C_{\alpha}} E_{\alpha}\left(-\frac{t^{\alpha}}{R C_{\alpha}}\right),
$$

where $q_{0 C_{\alpha}}$ denotes the initial value of $q_{C_{\alpha}}($ at $t=0)$ and $E_{\alpha}()$ stands for the Mittag-Leffler function [26] which can be defined in terms of arbitrary variable, $x$, as

$$
E_{\alpha}(x)=\sum_{k=0}^{\infty}\left[\frac{x^{k}}{\Gamma(\alpha k+1)}\right] .
$$

On the other hand, the charge of the conventional capacitor-resistor circuit, i.e., $q_{C}$, obeys

$$
\frac{d q_{C}}{d t}+\frac{q_{C}}{R C}=0
$$

Thus, it can be analytically given by

$$
q_{C}=q_{0 C} \exp \left(-\frac{t}{R C}\right)
$$

where $q_{0 C}$ denotes the initial value of $q_{C}$.

With (25), $q_{C_{\alpha}}$ can be simulated for various values of $\alpha$ by assuming that $q_{0 C_{\alpha}}=1, C_{\alpha}=0.01$, and $R=1$ as depicted in Figure 6 where the charge of the conventional capacitor-resistor circuit, i.e., $q_{C}$, simulated by using (28) has also been included. It can be seen from Figure 6 that the decreasing rate of $q_{C_{\alpha}}$, which is much faster than that of $q_{C}$ and referred to the discharging rate of the fractional capacitor, is inversely proportional to $\alpha$. Noted that the aforesaid relationship between the flux decaying rate of the fractional inductor and $\alpha$ can also be demonstrated in a similar manner.

\section{Breaking the Impasse Points of the Phase Portraits between Flux and Charge}

According to [9], the phase portraits between $\varphi$ and $q$ of the $\varphi$-controlled memristor-inductor circuit exhibit discontinuous jumps at the impasse points which can be broken by the effect of parasitic capacitance. By using intuition, it can be stated that such impasse points breaking of $q$-controlled memristor-capacitor circuit can be occurred by the parasitic inductor albeit this is not mentioned in [9]. In this work, we 


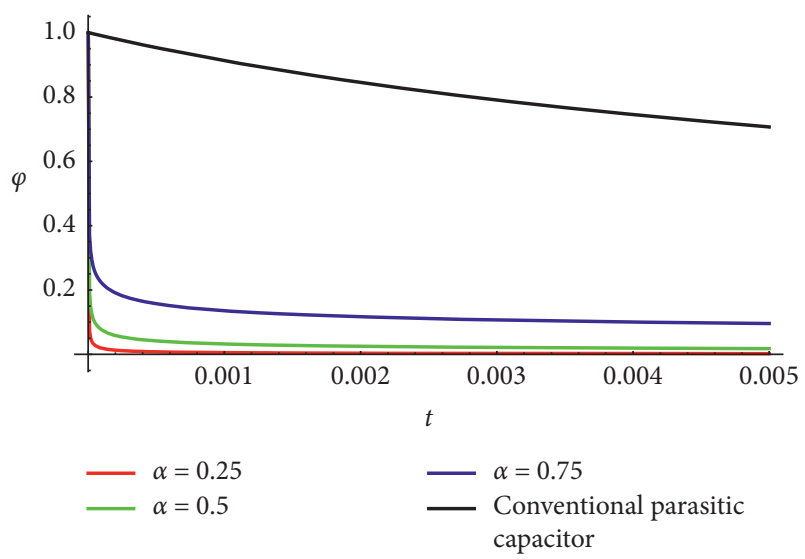

FIgURE 3: $\varphi$ vs $t$ of the memristor affected by the parasitic fractional capacitor and by the conventional parasitic capacitor [9].

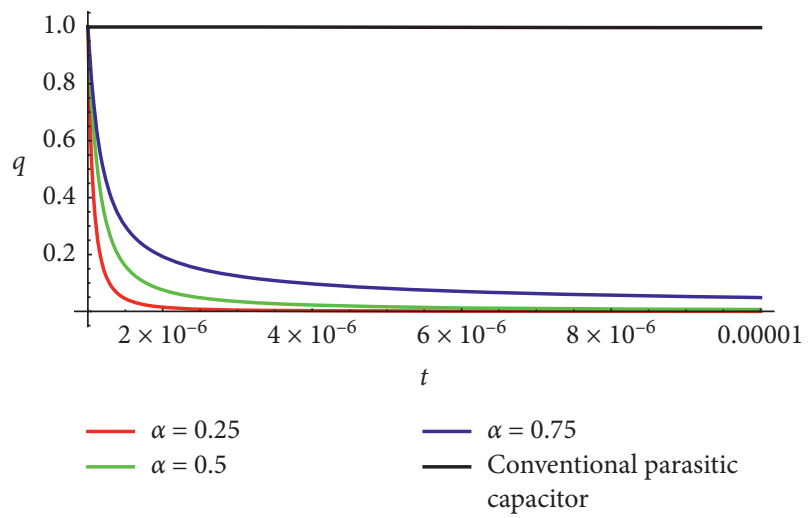

FIGURE 4: $q$ vs $t$ of the memristor affected by the parasitic fractional capacitor and by the conventional parasitic capacitor [9].

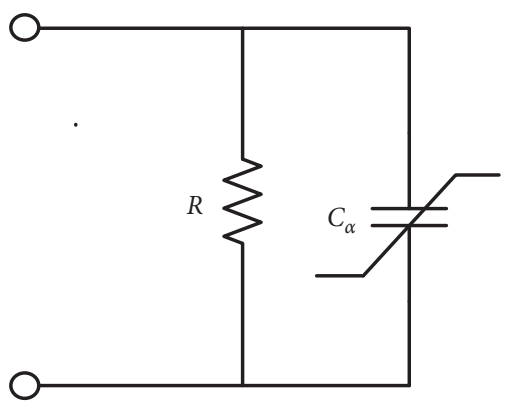

FIgURE 5: The source-free resistor-fractional capacitor circuit.

study the effect of the parasitic fractional element to the phase portraits between $\varphi$ and $q$ of the memristor-inductor/ capacitor circuits. Firstly, the memristor-inductor circuit affected by the parasitic fractional capacitor will be considered. Since we assume that the memristor and inductor are connected in parallel, the parasitic fractional capacitor included circuit can be modeled as depicted in Figure 7. Therefore, we have

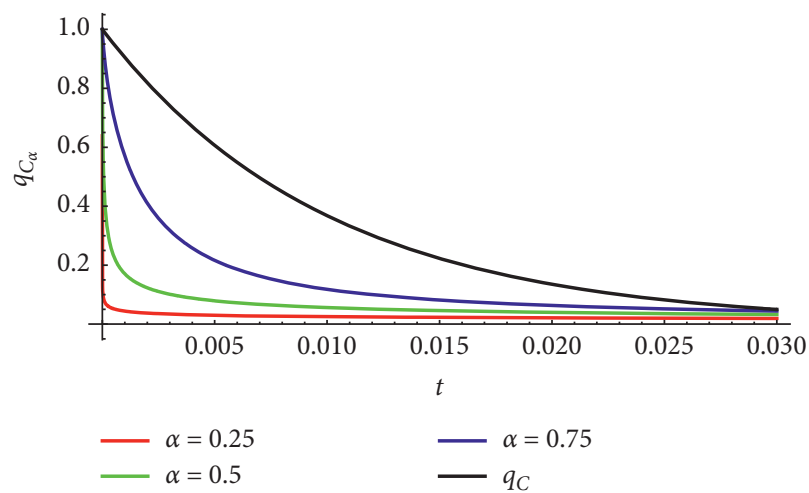

FIgURE 6: $q_{C_{\alpha}} / q_{C}$ vs $t$ of a source-free fractional capacitor-resistor circuit/a source-free conventional capacitor-resistor circuit.

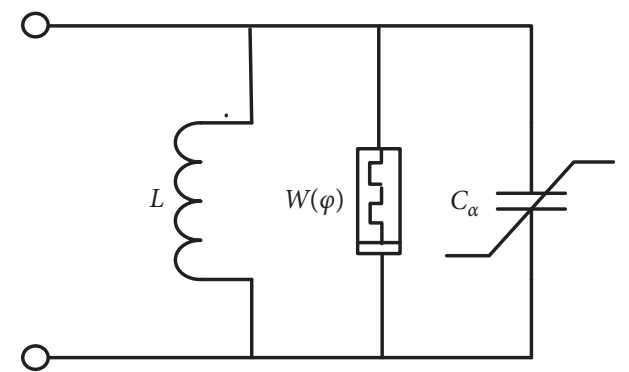

FIgURE 7: The memristor-inductor circuit with the parasitic fractional capacitor.

$$
\begin{gathered}
\frac{d Q_{L}}{d t}=-\frac{\varphi}{L}, \\
D^{\alpha}[\varphi]=\frac{Q_{L}-g(\varphi)}{C_{\alpha}},
\end{gathered}
$$

where $Q_{L}=-q_{L}$ which stands for the charge flowing through the inductor. Noted also that $Q_{L}$ depends on $q$ due to Kirchhoff's charge law (KCHL) [8] and we assume that the memristor is $\varphi$-controlled.

If we let

$$
g(\varphi)=\frac{\varphi^{3}}{3}-\varphi
$$

Equations (29) and (30) become

$$
\begin{aligned}
\frac{d Q_{L}}{d t} & =-\frac{\varphi}{L}, \\
D^{\alpha}[\varphi] & =\frac{Q_{L}-\left(\varphi^{3} / 3\right)+\varphi}{C_{\alpha}} .
\end{aligned}
$$

After applying the Adomian decomposition by keeping in mind that $d Q_{L} / d t \approx D^{0.999 \ldots}\left[Q_{L}\right]$ and the fractional integration of order $0.999 \ldots$ can be approximated by the integer one, we obtained

$$
\begin{aligned}
\varphi & =\varphi_{0}+\varphi_{1}+\varphi_{2} \cdots, \\
Q_{L} & =Q_{L 0}+Q_{L 1}+Q_{L 2} \cdots,
\end{aligned}
$$


where $Q_{L 0}$ denotes the initial value of $Q_{L}$ (at $\left.t=0\right)$. Noted also that

$$
\begin{aligned}
& \varphi_{1}=-\frac{t^{\alpha}\left(Q_{L 0}+\varphi_{0}-\left(\varphi_{0}^{3} / 3\right)\right)}{C_{\alpha} \Gamma(\alpha+1)}, \\
& \varphi_{2}=\frac{t^{\alpha}}{3 C_{\alpha}^{2}}\left[\frac{\left(\varphi_{0}^{2}-1\right)\left(\varphi_{0}^{3}-3 \varphi_{0}-3 Q_{L 0}\right) t^{\alpha}}{\Gamma(2 \alpha+1)}-\frac{3 C_{\alpha} \varphi_{0} t}{\Gamma(\alpha+2)}\right], \\
& \vdots
\end{aligned}
$$

$$
\begin{aligned}
Q_{L 1} & =-\frac{\varphi_{0} t}{L}, \\
Q_{L 2} & =\frac{t^{\alpha+1}\left(\varphi_{0}^{3}-3 \varphi_{0}-3 Q_{L 0}\right)}{3 L C_{\alpha} \Gamma(\alpha+2)} .
\end{aligned}
$$

For determining the numerical solution of (32) and (33), the integer derivative-based approximation of $D^{\alpha}[\varphi]$ by using the moments of a function has also been used. As a result, we have

$$
\begin{aligned}
& \frac{d Q_{L}}{d t}=-\frac{\varphi}{L} \\
& \frac{d \varphi}{d t}=\frac{\left(Q_{L} / C_{\alpha}\right)-\left(\varphi^{3} / 3 C_{\alpha}\right)+\left(\varphi / C_{\alpha}\right)+\sum_{p=2}^{N}\left(\Gamma(p-1+\alpha) t^{1-p-\alpha} V_{p}\right) /(\Gamma(2-\alpha) \Gamma(\alpha-1)(p-1) !)-\left(\left(1+\sum_{p=2}^{N}[(\Gamma(p-1+\alpha)) /(\Gamma(\alpha)(p-1) !)]\right) /(\Gamma(1-\alpha)) t^{-\alpha}\right)}{\left(\left(1+\sum_{p=1}^{N}(\Gamma(p-1+\alpha)) / \Gamma(\alpha-1) p !\right) /(\Gamma(2-\alpha)) t^{1-\alpha}\right.}, \\
& \frac{d V_{2}}{d t}=-\varphi \\
& \frac{d V_{3}}{d t}=-2 t \varphi, \\
& \quad \vdots \\
& \frac{d V_{N}}{d t}=(1-N) t^{N-2} \varphi .
\end{aligned}
$$

In [9] where the conventional parasitic capacitor has been considered on the other hand, the following conventional ODEs will be obtained instead [9]:

$$
\begin{aligned}
\frac{d Q_{L}}{d t} & =-\frac{\varphi}{L}, \\
\frac{d \varphi}{d t} & =\frac{Q_{L}-\left(\varphi^{3} / 3\right)+\varphi}{C_{\alpha}} .
\end{aligned}
$$

By using (38) with $N=7$, the numerical solution of a system of FDE composed of (32) and (33), i.e., $\varphi$ and $Q_{L}$, can be obtained and simulated against $t$ for various values of $\alpha$ as depicted in Figures 8 and 9 where $\varphi_{0}=0.1, Q_{L 0}=0.1$, $L=1$ [9], and $C_{\alpha}=0.01$ have also been assumed. Moreover, $\varphi$ and $Q_{L}$ of the memristor-inductor circuit affected by the conventional parasitic capacitor have also been included in these figures. Noted that the initial values of $V_{2}, V_{3}, \ldots, V_{N}$ have been automatically assumed to be 0 due to the adopted approximation of the fractional derivative. From these figures, it can be seen that both $\varphi$ and $Q_{L}$ of both the parasitic fractional capacitor and conventional parasitic capacitor-affected circuits exhibit the oscillations, i.e., the memristor-inductor circuit with parasitic capacitor/ fractional capacitor become an oscillator/a fractional oscillator. Noted that the simulation of the parasitic conventional capacitor-affected circuit studied in the previous work has been performed by using (39) and (40). As proposed in [9], the memristor-inductor circuit under the effect of the conventional parasitic capacitor becomes the conventional Van der Pol oscillator. In this work, it has been found that the parasitic fractional capacitor-affected memristor-inductor circuit becomes a fractional Van der Pol oscillator [27-29]. This is not surprising as (32) and (33) resemble the state space representation of such oscillator. We have also found that the amplitude of $Q_{L}$ and the oscillating frequencies of both $\varphi$ and $Q_{L}$ are affected by $\alpha$. This is also reasonable as the significant effect of the order on the output's magnitude and frequency is a typical characteristic of any fractional oscillator [30-32].

Now, consider the phase portraits between $\varphi$ and $Q_{L}$ which can be simulated for various values of $\alpha$ by also assuming that $\varphi_{0}=1$ and $C_{\alpha}=0.01$ as depicted in Figure 10 where the phase portrait of the conventional parasitic capacitor-affected memristor-inductor circuit proposed in [9] has also been included. Equation (38) has been used for the simulation of the circuit with the parasitic fractional capacitor where (39) and (40) have been adopted in this work in order to simulate the conventional parasitic capacitor-affected circuit for comparison. Similarly to that of the circuit affected by the conventional parasitic capacitor, there is none of any impasse point on each phase portrait of the memristor-inductor circuit with the parasitic fractional 


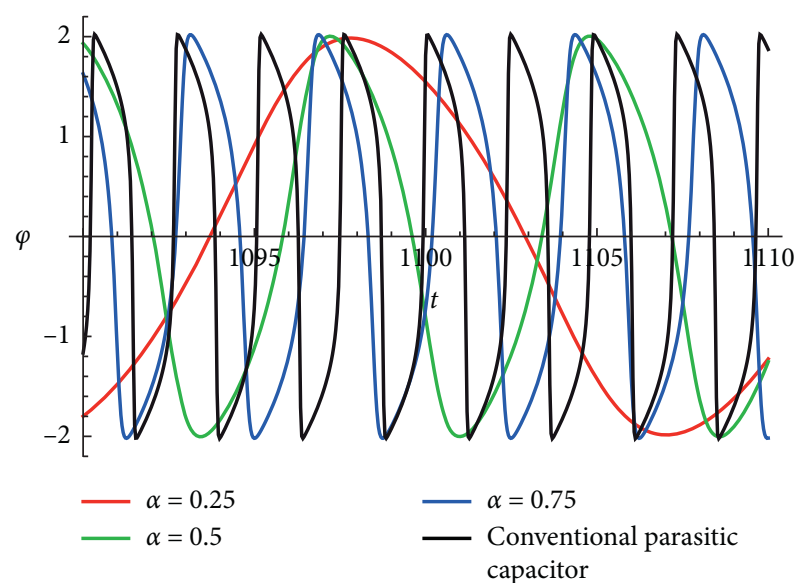

FIgURE 8: $\varphi$ vs $t$ of the memristor-inductor circuit with the parasitic fractional capacitor/conventional parasitic capacitor.

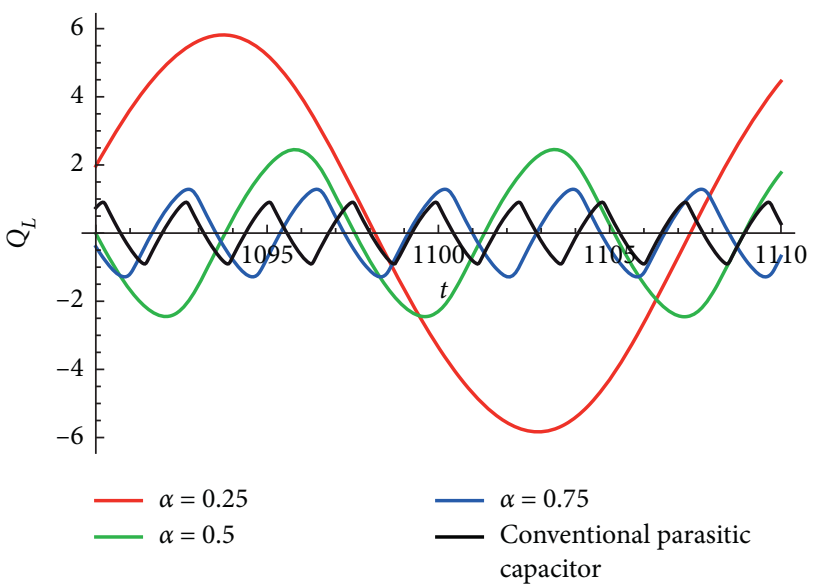

Figure 9: $Q_{L}$ vs $\varphi$ of the memristor-inductor circuit with the parasitic fractional capacitor/conventional parasitic capacitor [9].

capacitor as there is no jump behavior occurred. Therefore, it can be stated that such impasse points on the phase portraits can also be broken by the effect of the parasitic fractional capacitor as well as the conventional parasitic capacitor [9] despite its fractional order. Such impasse point breakings yield the continuous oscillations of $\varphi$ and $Q_{L}$ as shown in Figures 8 and 9. Moreover, it has been found that $\alpha$ significantly affects both size and shape of the phase portraits. This is because the memristor-inductor circuit with the parasitic fractional capacitor becomes a fractional Van der Pol oscillator which the significant effects of $\alpha$ on the phase portraits between outputs are typical $[27,28]$, as stated above. As expected, the simulated phase portrait with $\alpha$ approaching 1 becomes more similar to that of the memristor-inductor circuit with the conventional parasitic capacitor. This is not surprising as the charge-flux relationship of the fractional capacitor with $\alpha=1$ is similar to that of the conventional capacitor as can be seen from (7). From the depicted phase portraits, it can also be seen that the memory and the linearity of the memristor-inductorparasitic fractional capacitor circuit are, respectively, inversely and directly proportional to $\alpha$.
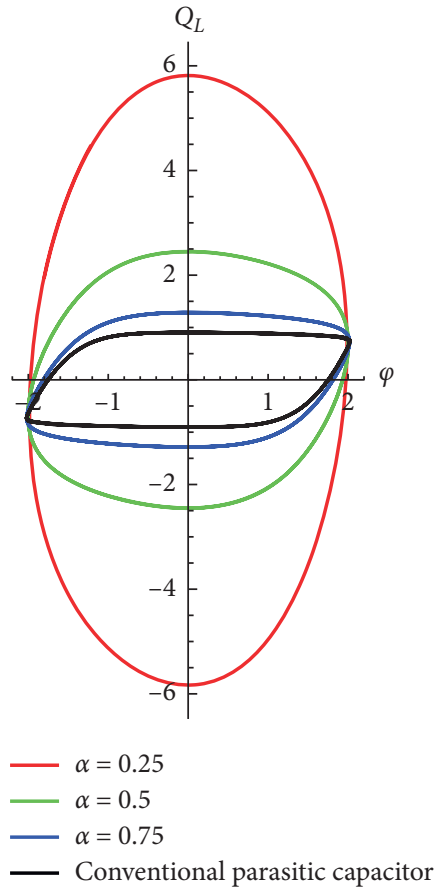

FIgURE 10: $Q_{\mathrm{L}}$ vs $\varphi$ of the memristor-inductor circuit with the parasitic fractional capacitor/conventional parasitic capacitor [9].

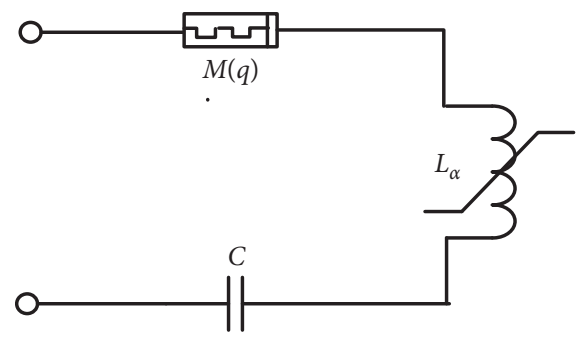

FIgURE 11: The memristor-capacitor circuit with parasitic fractional inductor.

At this point, we will analyze the effects of the parasitic fractional inductor to the memristor-capacitor circuit. Since we assume that the memristor and capacitor are connected in parallel, the parasitic fractional inductor included circuit can be modeled as depicted in Figure 11. As a result, the system of FDE given by (41) and (42) can be obtained if we let the memristor be of a $q$-controlled type with $f(q)=\left(q^{3} / 3\right)-q$.

$$
\begin{aligned}
\frac{d \Phi_{C}}{d t} & =-\frac{q}{C} \\
D^{\alpha}[q] & =\frac{\Phi_{C}-\left(q^{3} / 3\right)+q}{L_{\alpha}} .
\end{aligned}
$$

Noted that $\Phi_{C}=\varphi_{C}$ which denotes the flux of the capacitor, and $\Phi_{C}$ depends on $\varphi$ due to Kirchhoff's flux law (KFLL) [8]. By solving (41) and (42) analytically with the Adomian decomposition, we have 


$$
\begin{aligned}
q & =q_{0}+q_{1}+q_{2} \cdots, \\
\Phi_{C} & =\Phi_{C 0}+\Phi_{C 1}+\Phi_{C 2} \cdots
\end{aligned}
$$

where $\Phi_{C 0}$ denotes the initial value of $\Phi_{C}($ at $t=0)$ and

$$
\begin{aligned}
& q_{1}=-\frac{t^{\alpha}\left(\Phi_{C 0}+q_{0}-\left(q_{0}^{3} / 3\right)\right)}{L_{\alpha} \Gamma(\alpha+1)}, \\
& q_{2}=\frac{t^{\alpha}}{3 L_{\alpha}^{2}}\left[\frac{\left(q_{0}^{2}-1\right)\left(q_{0}^{3}-3 q_{0}-3 \Phi_{C 0}\right) t^{\alpha}}{\Gamma(2 \alpha+1)}-\frac{3 L_{\alpha} q_{0} t}{\Gamma(\alpha+2)}\right] \\
& \vdots \\
& \Phi_{C 1}=-\frac{q_{0} t}{C}, \\
& \Phi_{C 2}=\frac{t^{\alpha+1}\left(q_{0}^{3}-3 q_{0}-3 \Phi_{C 0}\right)}{3 C L_{\alpha} \Gamma(\alpha+2)} . \\
& \vdots
\end{aligned}
$$

For determining $q$ and $\Phi_{C}$ numerically, the integer derivative-based approximation of $D^{\alpha}[q]$ by using the moments of a function with $N=7$ has been used. Besides other initial values which have been assumed to be 0 due to the adopted approximation of $D^{\alpha}[q]$, we let $q_{0}=0.1, \Phi_{C 0}$ $=0.1, C=1$, and $L_{\alpha}=0.01$. The resulting $q$ and $\Phi_{C}$ and their phase portraits have been simulated for various values of $\alpha$ as depicted in Figures 12-14 where those of the conventional parasitic inductor-affected circuit have also been included. It should be mentioned here that the conventional parasitic inductor-related results can be simulated by using the following ODEs:

$$
\begin{aligned}
\frac{d \Phi_{C}}{d t} & =-\frac{q}{C}, \\
\frac{d q}{d t} & =\frac{\Phi_{C}-\left(q^{3} / 3\right)+q}{L} .
\end{aligned}
$$

From Figures 12 and 13, $\alpha$-controlled continuous oscillations of both $q$ and $\Phi_{C}$ can be observed for the circuit with the parasitic fractional inductor. This is because the entire circuit acts as a fractional oscillator. From the phase portraits between $q$ and $\Phi_{C}$ depicted in Figure 14, it can be observed that the impasse points on the phase portraits can also be broken by the parasitic fractional inductor similarly to the conventional parasitic inductor. It can also be seen that $\alpha$ employs significant effects to both size and shape of the phase portrait of the parasitic fractional inductor-affected circuit as such circuit becomes a fractional Van der Pol oscillator. Moreover, the phase portrait of the parasitic fractional inductor-affected circuit with $\alpha$ approaching 1 becomes more similar to that of the circuit with conventional parasitic inductor which is actually the memristorcapacitor-inductor-based Van der Pol oscillator [8]. This is because the fractional inductor with $\alpha=1$ employs a

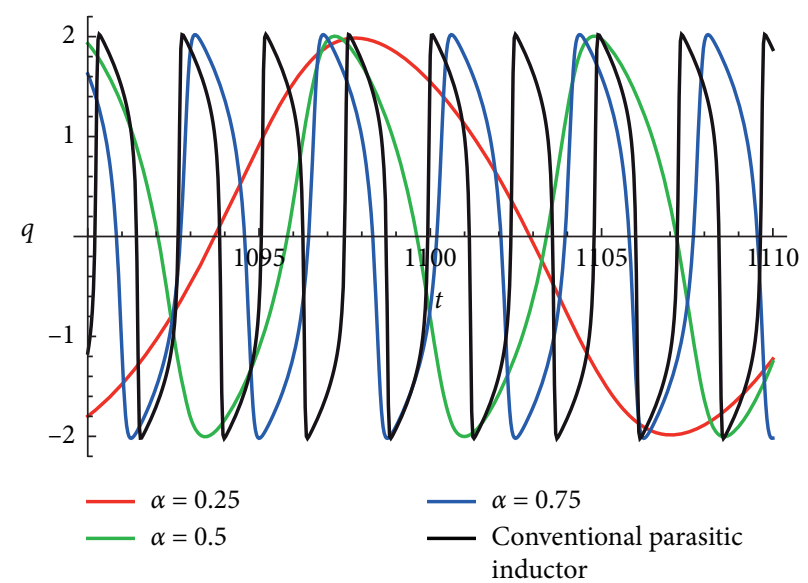

Figure 12: $: q$ vs $t$ of the memristor-capacitor circuit with the parasitic fractional inductor/conventional parasitic inductor.

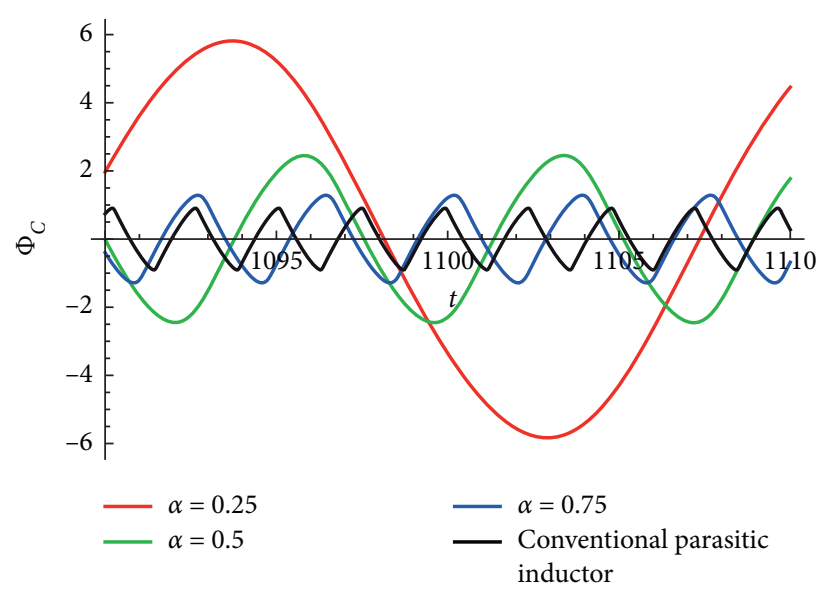

Figure 13: $\Phi_{C}$ vs $t$ the memristor-capacitor circuit with the parasitic fractional inductor/conventional parasitic inductor.

similar charge-flux relationship to that of the conventional inductor as can be seen from (6).

\section{Nonlinear Effect of the Parasitic Fractional Element}

The conventional parasitic element can be nonlinear [9] and so does the parasitic fractional element. Since the ODEs of the memristor-inductor circuit affected by the nonlinear conventional parasitic capacitor can be given by [9]

$$
\begin{aligned}
\frac{d Q_{L}}{d t} & =-\frac{\varphi}{L}, \\
\frac{d \varphi}{d t} & =\frac{Q_{L}-g(\varphi)}{C(\varphi)},
\end{aligned}
$$

where $C(\varphi)$ which is a function of $\varphi$ is the capacitance of the nonlinear conventional parasitic capacitor, the system 


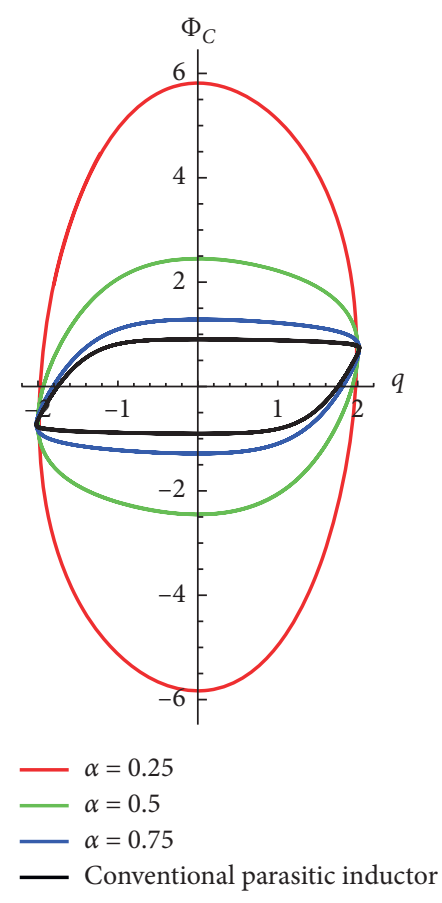

FIgURE 14: $\Phi_{C}$ vs $q$ the memristor-capacitor circuit with the parasitic fractional inductor/conventional parasitic inductor.

of FDE of the memristor-inductor circuit affected by nonlinear parasitic fractional capacitor can be given as follows:

$$
\begin{aligned}
\frac{d Q_{L}}{d t} & =-\frac{\varphi}{L}, \\
D^{\alpha}[\varphi] & =\frac{Q_{L}-g(\varphi)}{C_{\alpha}(\varphi)},
\end{aligned}
$$

where $C_{\alpha}(\varphi)$ which is a function of $\varphi$ stands for the pseudocapacitance of the nonlinear parasitic fractional capacitor. Noted also that $\varphi_{C_{\alpha}}=\varphi$ due to the circuit topology.

By assuming that $C_{\alpha}(\varphi)=C_{\alpha}\left(1+\varphi^{2}\right)$ where $C_{\alpha}$ means $C_{\alpha}(0)$ in this scenario and using the Adomian decomposition, the analytical solution of (51) and (52) can also be given by (34) and (35) but with the following terms instead of those given by (36) and (37).

$$
\begin{aligned}
\varphi_{1}= & -\frac{t^{\alpha}\left(Q_{L 0}+\varphi_{0}-\left(\varphi_{0}^{3} / 3\right)\right)}{C_{\alpha}\left(1+\varphi_{0}^{2}\right) \Gamma(\alpha+1)}, \\
\varphi_{2}= & \frac{t^{\alpha}}{9 C_{\alpha}^{2}\left(1+\varphi_{0}^{2}\right)^{3}}\left[\frac{\left(\varphi_{0}^{4}+6 \varphi_{0}^{2}+6 \varphi_{0} Q_{L 0}-3 Q_{L 0}\right) t^{\alpha}}{\left(\varphi_{0}^{3}-3 \varphi_{0}-3 Q_{L 0}\right)^{-1} \Gamma(2 \alpha+1)}\right. \\
& \left.-\frac{9 C_{\alpha} \varphi_{0} t\left(1+\varphi_{0}^{2}\right)^{2}}{L \Gamma(\alpha+2)}\right],
\end{aligned}
$$

$$
\begin{aligned}
Q_{L 1} & =-\frac{\varphi_{0} t}{L} \\
Q_{L 2} & =\frac{t^{\alpha+1}\left(\varphi_{0}^{3}-3 \varphi_{0}-3 Q_{L 0}\right)}{3 L C_{\alpha}\left(1+\varphi_{0}^{2}\right) \Gamma(\alpha+2)} .
\end{aligned}
$$

For determining the numerical solution of (51) and (52), the integer derivative-based approximation of $D^{\alpha}[\varphi]$ by using the moments of a function with $N=7$ has been adopted and $\varphi_{0}=0.1, Q_{L 0}=0.1, L=1$, and $C_{\alpha}=0.01$ have also been assumed. The other initial values have been assumed to be 0 by default according to the adopted approximation of $D^{\alpha}[\varphi]$. The obtained solutions, i.e., $\varphi$ and $Q_{L}$, and their phase portraits have been simulated for various values of $\alpha$ as depicted in Figures 15-17 where the nonlinear conventional parasitic capacitor-related results including the phase portraits [9] simulated here by using (49) and (50) with $C(\varphi)=$ $C(0)\left(1+\varphi^{2}\right)$ have also been included. From Figures 15 and 16 , it can be seen that the nonlinear parasitic fractional capacitor also yields the continuous oscillations of $\varphi$ and $Q_{L}$ similarly to the nonlinear conventional parasitic capacitor as reported by [9]. Moreover, the properties of the oscillation caused by the nonlinear parasitic fractional capacitor are $\alpha$ dependent similarly to those caused by its linear counterpart.

From Figure 17, it can be observed that the nonlinear parasitic fractional capacitor also causes the impasse points breaking of the phase portraits between $\varphi$ and $Q_{L}$ similarly to the nonlinear conventional parasitic capacitor as also reported by [9]. Noted that both sizes and shapes of the phase portraits of the circuit with the nonlinear parasitic fractional capacitor are also significantly affected by $\alpha$ despite its nonlinearity. This is not surprising because the dependency on $\alpha$ of the phase portraits between outputs can be typically found in any nonlinear fractional system [33, 34] including our memristor-inductornonlinear parasitic fractional capacitor circuit. Moreover, it can be seen that the phase portraits between $\varphi$ and $Q_{L}$ depicted in Figure 17 have wider loop areas which refers to less linearity and more memory, than those of the previous case depicted in Figure 6. Such wider loop areas can be clearly observed when $\alpha$ approaches 1 . On the other hand, the phase portraits depicted in Figure 12 also have more roundish shapes compared to those depicted in Figure 10 when $\alpha$ approaches 1 . These differences in the sizes and shapes of the phase portraits are caused by the nonlinearities which have been taken into account in this scenario. Therefore, it can be seen that the parasitic fractional capacitor also has the nonlinear effect similarly to the conventional parasitic capacitor but with dependency on $\alpha$. When $\alpha$ approaches 1, such effect yields merely a little loop area enlargement plus more roundish phase portraits similarly to the nonlinear effect of the conventional parasitic capacitor [9]. When $\alpha$ approaches 0 , the nonlinear effect of the parasitic fractional capacitor yields the significant enlargement in the loop area. Therefore, it can be stated that the nonlinear effect of the parasitic element also exists in the fractional order domain where the fractional element can be assumed. 


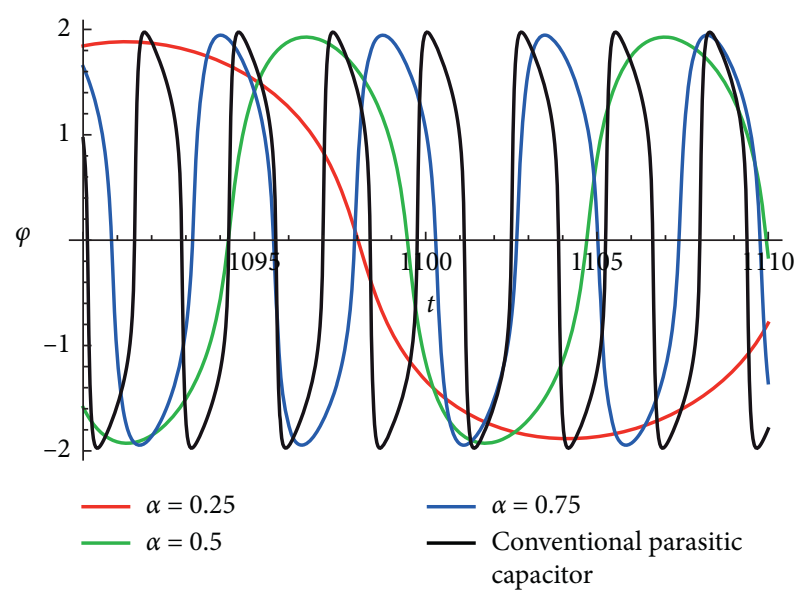

FIgURE 15: $\varphi$ vs $t$ of the memristor-inductor circuit with the nonlinear parasitic fractional capacitor/nonlinear conventional parasitic capacitor.

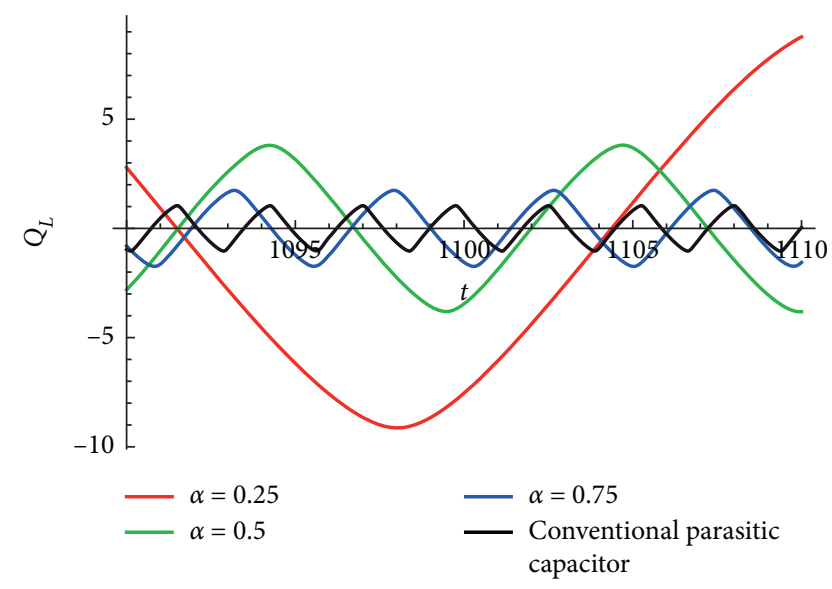

Figure 16: $Q_{L}$ vs $t$ of the memristor-inductor circuit with the nonlinear parasitic fractional capacitor/nonlinear conventional parasitic capacitor.

Now we let the parasitic fractional inductor be nonlinear. With the nonlinear conventional parasitic inductor, the dynamic of memristor-capacitor circuit can be analytically described by the following ODEs:

$$
\begin{aligned}
\frac{d \Phi_{C}}{d t} & =-\frac{q}{C}, \\
\frac{d q}{d t} & =\frac{\Phi_{C}-f(q)}{L(q)},
\end{aligned}
$$

where $L(q)$ is the inductance of the nonlinear parasitic inductor.

Therefore, the system of FDE of the memristor-capacitor circuit affected by such nonlinear parasitic fractional inductor can be given as follows:

$$
\begin{aligned}
\frac{d \Phi_{C}}{d t} & =-\frac{q}{C} \\
D^{\alpha}[q] & =\frac{\Phi_{C}-f(q)}{L_{\alpha}(q)},
\end{aligned}
$$

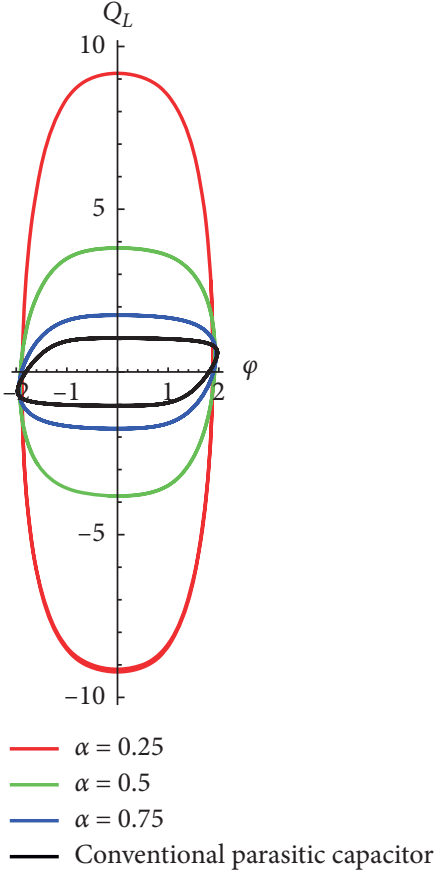

Figure 17: $Q_{L}$ vs $\varphi$ of the memristor-inductor circuit with the nonlinear parasitic fractional/nonlinear conventional parasitic capacitor [9].

where $L_{\alpha}(q)$, which is a function of $q$, denotes the inductivity of the nonlinear parasitic fractional inductor. Noted also that $q_{L_{\alpha}}=q$ due to the circuit topology.

If we let $L_{\alpha}(q)=L_{\alpha}\left(1+q^{2}\right)$ where $L_{\alpha}=L_{\alpha}(0)$, both $q$ and $\Phi_{C}$ can also be analytically given by (43) and (44) but with

$$
q_{1}=-\frac{t^{\alpha}\left(\Phi_{C 0}+q_{0}-\left(q_{0}^{3} / 3\right)\right)}{L_{\alpha}\left(1+q_{0}^{2}\right) \Gamma(\alpha+1)},
$$$$
q_{2}=\frac{t^{\alpha}}{9 L_{\alpha}^{2}\left(1+q_{0}^{2}\right)^{3}}\left[\frac{\left(q_{0}^{4}+6 q_{0}^{2}+6 q_{0} \Phi_{C 0}-3 \Phi_{C 0}\right) t^{\alpha}}{\left(q_{0}^{3}-3 q_{0}-3 \Phi_{C 0}\right)^{-1} \Gamma(2 \alpha+1)}\right.
$$

$$
\left.-\frac{9 L_{\alpha} q_{0} t\left(1+q_{0}^{2}\right)^{2}}{C \Gamma(\alpha+2)}\right]
$$

$\vdots$

$$
\begin{aligned}
\Phi_{C 1} & =-\frac{q_{0} t}{C}, \\
\Phi_{C 2} & =\frac{t^{\alpha+1}\left(q_{0}^{3}-3 q_{0}-3 \Phi_{C 0}\right)}{3 C L_{\alpha}\left(1+q_{0}^{2}\right) \Gamma(\alpha+2)} .
\end{aligned}
$$

after applying the Adomian decomposition.

By numerically solving (57) and (58) via the moments of a function-based integer derivative-based approximation of $D^{\alpha}[q]$ with $N=7$ where $q_{0}=0.1, \Phi_{C 0}=0.1, C=1$, and $L_{\alpha}=0.01$ have been assumed, $q, \Phi_{C}$, and their phase portraits have been simulated for various values of $\alpha$ as depicted in Figures 18-20 where the nonlinear conventional parasitic inductor-related results have also been included for 


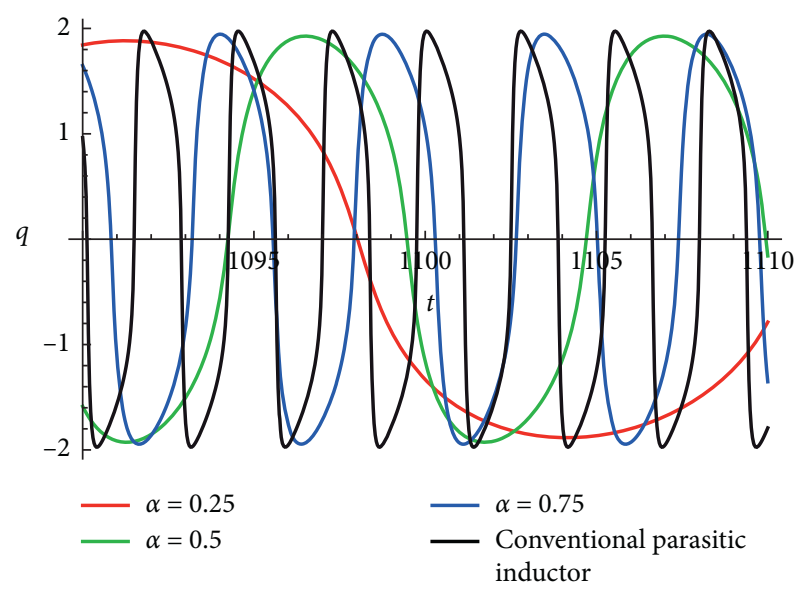

FIgURE 18: $q$ vs $t$ of the memristor-capacitor circuit with the nonlinear parasitic fractional inductor/nonlinear conventional parasitic inductor.

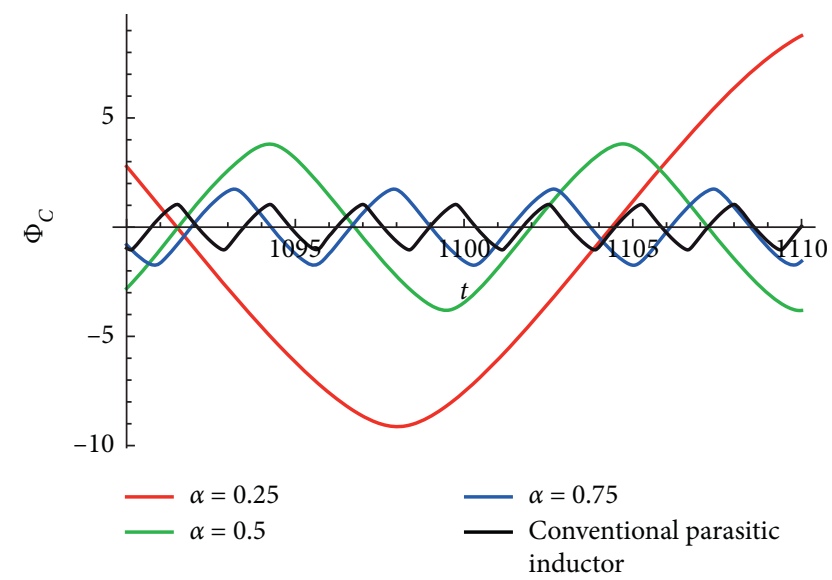

Figure 19: $\Phi_{C}$ vs $t$ the memristor-capacitor circuit with the nonlinear parasitic fractional inductor/nonlinear conventional parasitic inductor.

comparison. Noted also that other initial values are automatically assumed to be 0 due to the adopted approximation of $D^{\alpha}[q]$. Moreover, (55) and (56) with $L(q)=L(0)\left(1+q^{2}\right)$ have been used for simulating such nonlinear conventional parasitic inductor-related results.

From these figures, the continuous oscillations of $q$ and $\Phi_{C}$ along with the impasse points breaking of the phase portraits between $q$ and $\Phi_{C}$ can be observed from both nonlinear conventional parasitic inductor and nonlinear fractional parasitic inductor-related results albeit the properties of the latter are $\alpha$ dependent because our memristor-capacitor-nonlinear parasitic fractional inductor circuit acts as a nonlinear fractional oscillator which is a nonlinear fractional system. Therefore, it can be seen that the parasitic fractional inductor also employs the nonlinear effect similarly to the conventional parasitic inductor but with dependency on $\alpha$. By this and the aforementioned nonlinear effect of the parasitic fractional capacitor, the existence of the nonlinear effect of parasitic element in the

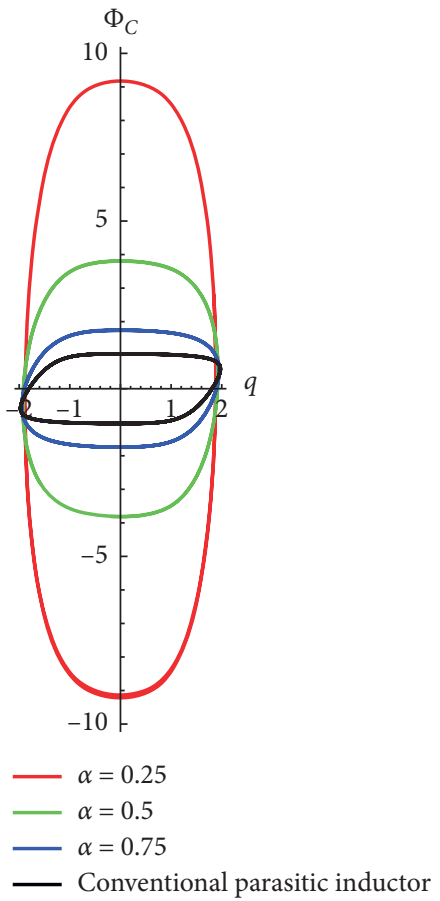

Figure 20: $\Phi_{C}$ vs $q$ the memristor-capacitor circuit with the nonlinear parasitic fractional inductor/nonlinear conventional parasitic inductor.

fractional order domain has been validated. The phase portraits between $q$ and $\Phi_{C}$ with wider loop areas compared to those depicted in Figure 14 can be seen where the significant loop area enlargement can be observed when $\alpha$ approaches 0 and more roundish shapes of the phase portraits can also be found when $\alpha$ approaches 1 . This is because the formerly neglected nonlinearities have been now considered.

\section{Conclusion}

The dynamic of the memristor under the effects of the parasitic fractional elements has been studied in this work based on the FDE approach where both $\varphi$-controlled and $q$ controlled memristors have been considered. The comparison to the previous work which studied such device affected by the parasitic conventional elements [9] has also been made. The analytical solutions of the formulated FDEs have been determined by using the Adomian decomposition method where the numerical solutions have been obtained by using the integer derivative-based approximation of the fractional derivative with the moments of a function. From the obtained solutions and simulations, we have found that the decaying of $\varphi$ and $q$ and the impasse point breaking of the phase portraits are also occurred by the effect of the parasitic fractional elements as well as the parasitic conventional elements as reported by [9]. It has also been found that the decaying rates of $\varphi$ and $q$ are inversely proportional to $\alpha$ which also affects both size and shape of phase portraits and the properties of the oscillatory outputs of the memristor-based circuits which acts as the fractional Van 
der Pol oscillators. Moreover, we have found that the memory and the linearity are, respectively, inversely and directly proportional to $\alpha$ and the nonlinear effect of parasitic element, which occurs in the conventional integer domain, remains valid in the fractional order domain where the influence of such effect is $\alpha$ dependent. From these results, it can be seen that this research gives a further understanding on the characteristics of memristor which is a state-of-the-art electrical circuit element. Since this work limitedly focuses on the theoretical memristor similarly to [9], a similar study on the practical memristor, e.g., NTC/ PTC thermistor [35] and HP memristor, which also suffers the adverse effects of parasitic elements as reported in previous studies, e.g., [35-37], should be considered as a tentative future work.

\section{Data Availability}

The simulated data used to support the findings of this study are included within the article.

\section{Conflicts of Interest}

The author declares that there are no conflicts of interest regarding the publication of this article.

\section{Acknowledgments}

The author would like to acknowledge Mahidol University, Thailand, for the online database service which is our primary information resource.

\section{References}

[1] L. Chua, "Memristor-the missing circuit element," IEEE Transactions on Circuit Theory, vol. 18, no. 5, pp. 507-519, 1971.

[2] D. B. Strukov, G. S. Snider, D. R. Stewart, and R. S. Williams, "The missing memristor found," Nature, vol. 453, no. 7191, pp. 80-83, 2008.

[3] A. Talukdar, A. G. Radwan, and K. N. Salama, "Generalized model for memristor-based Wien family oscillators," $M i$ croelectronics Journal, vol. 42, no. 9, pp. 1032-1038, 2011.

[4] Y. V. Pershin and M. Di Ventra, "Experimental demonstration of associative memory with memristive neural networks," Neural Networks, vol. 23, no. 7, pp. 881-886, 2010.

[5] B. L. Mouttet, "Programmable crossbar signal processor," US Patent 7302513, United States Patent and Trademark Office, Alexandria, VA, USA, 2007.

[6] J. H. Young, J. Y. Lee, S. Y. Choi, and J. W. Kim, "Microscopic origin of bipolar resistive switching of nano scale titaniumoxide thin films," Applied Physics Letters, vol. 95, no. 16, article 162108, 2009.

[7] M. Itoh and L. O. Chua, "Dynamics of memristor circuits," International Journal of Bifurcation and Chaos, vol. 24, no. 5, article 1430015, 2014.

[8] M. Itoh and L. O. Chua, "Duality of memristor circuits," International Journal of Bifurcation and Chaos, vol. 23, no. 1, p. 1330001, 2013.

[9] M. Itoh and L. O. Chua, "Parasitic effects on memristor dynamics," International Journal of Bifurcation and Chaos, vol. 26, no. 6, article 1630014, 2016.
[10] M. Itoh and L. O. Chua, "Memristor hamiltonian circuits," International Journal of Bifurcation and Chaos, vol. 21, no. 9, pp. 2395-2425, 2011.

[11] M. Nakagawa and K. Sorimachi, "Basic characteristics of a fractance device," IEICE Transaction on Fundamental of Electronics, Communications and Computer Sciences, vol. E75A, no. 12, pp. 1814-1819, 1992.

[12] M. Sugi, Y. Hirano, Y. F. Miura, and K. Saito, "Simulation of fractal immittance by analog circuits: an approach to the optimized circuits," IEICE Transaction on Fundamental of Electronics, Communications and Computer Sciences, vol. E82A, no. 8, pp. 1627-1634, 1999.

[13] B. T. Krishna, K. V. V. S. Reddy, and S. Santha Kumari, "Time domain response calculations of fractance device of order 1/2," Journal of Active and Passive Electronic Device, vol. 3, no. 3-4, pp. 355-367, 2008.

[14] C. Tang, F. You, G. Cheng, D. Gao, F. Fu, and X. Dong, "Modeling the frequency dependence of the electrical properties of the live human skull," Physiological Measurement, vol. 30, no. 12, pp. 1293-1301, 2009.

[15] A. Charef, "Analogue realisation of fractional-order integrator, differentiator and fractional $\mathrm{PI} \lambda \mathrm{D} \mu$ controller," IEE Proceedings-Control Theory and Applications, vol. 153, no. 6, pp. 714-720, 2006.

[16] L. Dork, J. Terpk, I. Petr, and F. Dorkov, "Electronic realization of the fractional-order systems," Acta Montanistica Slovaca, vol. 12, pp. 231-237, 2007.

[17] M. F. M. Lima, J. A. T. Machado, and M. Cris'ostomo, "Experimental signal analysis of robot impacts in a fractional calculus perspective," JACIII, vol. 11, pp. 1079-1085, 2007.

[18] R. Panda and M. Dash, "Fractional generalized splines and signal processing," Signal Processing, vol. 86, no. 9, pp. 2340-2350, 2006.

[19] H. Jafari and V. Daftardar-Gejji, "Solving a system of nonlinear fractional differential equations using Adomian decomposition," Journal of Computational and Applied Mathematics, vol. 196, no. 2, pp. 644-651, 2006.

[20] S. Pooseh, R. Almeida, and D. F. M. Torres, "Numerical approximations of fractional derivatives with applications," Asian Journal of Control, vol. 15, no. 3, pp. 698-712, 2013.

[21] L. O. Chua, "The fourth element," Proceedings of the IEEE, vol. 100, no. 6, pp. 1920-1927, 2012.

[22] L. O. Chua, Introduction to Nonlinear Network Theory, McGraw-Hill, Newyork, NY, USA, 1969.

[23] R. Banchuin, "Novel expressions for time domain responses of fractance device," Cogent Engineering, vol. 4, no. 1, article 1320823, 2017.

[24] I. Schäfer and K. Krüger, "Modelling of coils using fractional derivatives," Journal of Magnetism and Magnetic Materials, vol. 307, no. 1, pp. 91-98, 2006.

[25] T. J. Freeborn, B. Maundy, and A. S. Elwakil, "Measurement of supercapacitor fractional-order model parameters from voltage-excited step response," IEEE Journal on Emerging and Selected Topics in Circuits and Systems, vol. 3, no. 3, pp. $367-376,2013$.

[26] A. M. Mathai and H. J. Haubold, Special Functions for Applied Scientists, Springer, Newyork, NY, USA, 2010.

[27] V. Mishra, S. Das, H. Jafari, and S. H. Ong, "Study of fractional order van der Pol equation," Journal of King Saud University-Science, vol. 28, no. 1, pp. 55-60, 2016.

[28] R. S. Barbosa, J. T. Machado, I. M. Ferreira, and J. K. Tar, "Dynamics of the fractional-order Van der Pol oscillator," in Proceedings 2004. ICCC 2004. Second IEEE International 
Conference on Computational Cybernetics, pp. 373-378, IEEE, Vienna, Austria, August 2004.

[29] Y.-J. Shen, P. Wei, and S.-P. Yang, "Primary resonance of fractional-order van der pol oscillator," Nonlinear Dynamics, vol. 77, no. 4, pp. 1629-1642, 2014.

[30] A. G. Radwan, A. S. Elwakil, and A. M. Soliman, "Fractionalorder sinusoidal oscillators: design procedure and practical examples," IEEE Transactions on Circuits and Systems I: Regular Papers, vol. 55, no. 7, pp. 2051-2063, 2008.

[31] A. G. Radwan, A. M. Soliman, and A. S. Elwakil, "Design equations for fractional-order sinusoidal oscillators: four practical circuit examples," International Journal of Circuit Theory and Applications, vol. 36, no. 4, pp. 473-492, 2008.

[32] A. Soltan, A. G. Radwan, and A. M. Soliman, "General procedure for two integrator loops fractional order oscillators with controlled phase difference," in Proceedings of the 2013 25th International Conference on Microelectronics (ICM), pp. 1-4, IEEE, Beirut, Lebanon, December 2013.

[33] V. Vafaei, H. Kheiri, M. Javadi, and A.J. Akbarfam, "Classification of phase portraiats of linear and nonlinear fractional order systems," Journal of Fractional Calculus and Applications, vol. 8, no. 2, pp. 40-67, 2017.

[34] M. Rivero, J. J. Trujillo, and M. P. Velasco, "On deterministic fractional models," in New Trends in Nanotechnology and Fractional Calculus Applications, pp. 123-150, Springer, Dordrecht, Netherlands, 2010.

[35] M. P. Sah, C. Yang, H. Kim, B. Muthuswamy, J. Jevtic, and L. Chua, "A generic model of memristors with parasitic components," IEEE Transactions on Circuits and Systems I: Regular Papers, vol. 62, no. 3, pp. 891-898, 2015.

[36] B. Garda and M. J. Ogorzałek, "Modelling the generic $\mathrm{TiO}_{2}$ memristor with the parasitic components," in Proceedings of the 2016 International Conference on Signals and Electronic Systems (ICSES), pp. 173-176, IEEE, Kraków, Poland, September 2016.

[37] X. Yang, A. Adeyemo, A. Bala, and A. Jabir, "Parasitic effects on memristive logic architecture," in Proceedings of the 2017 27th International Symposium on Power and Timing Modeling, Optimization and Simulation (PATMOS), pp. 1-5, IEEE, Thessaloniki, Greece, September 2017. 


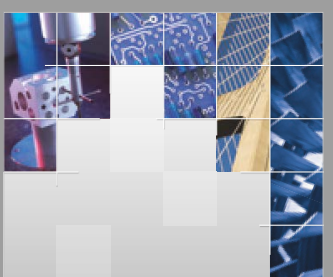

\section{Enfincering}
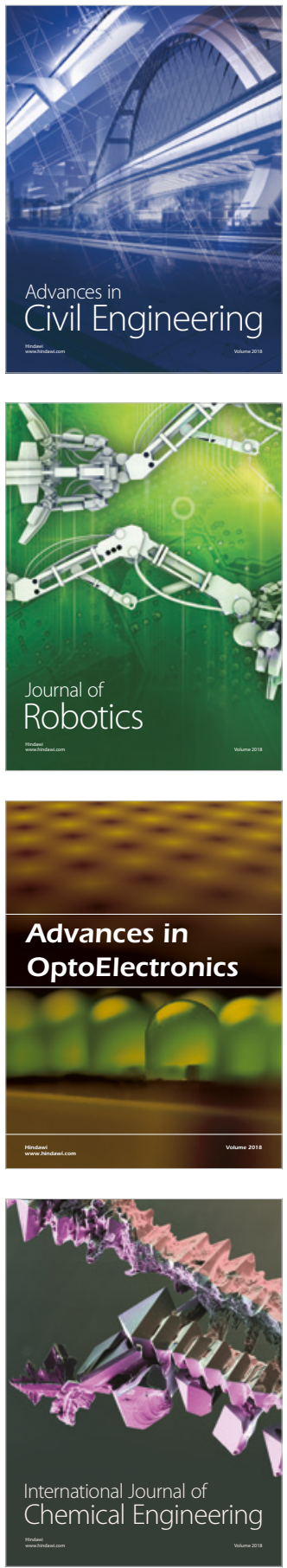

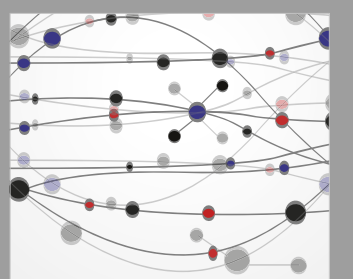

\section{Rotating \\ Machinery}

The Scientific World Journal

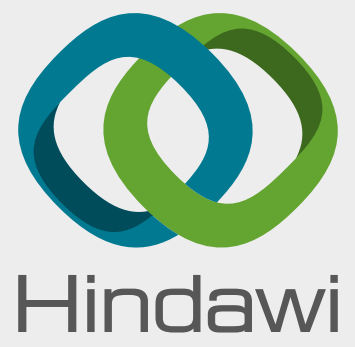

Submit your manuscripts at

www.hindawi.com
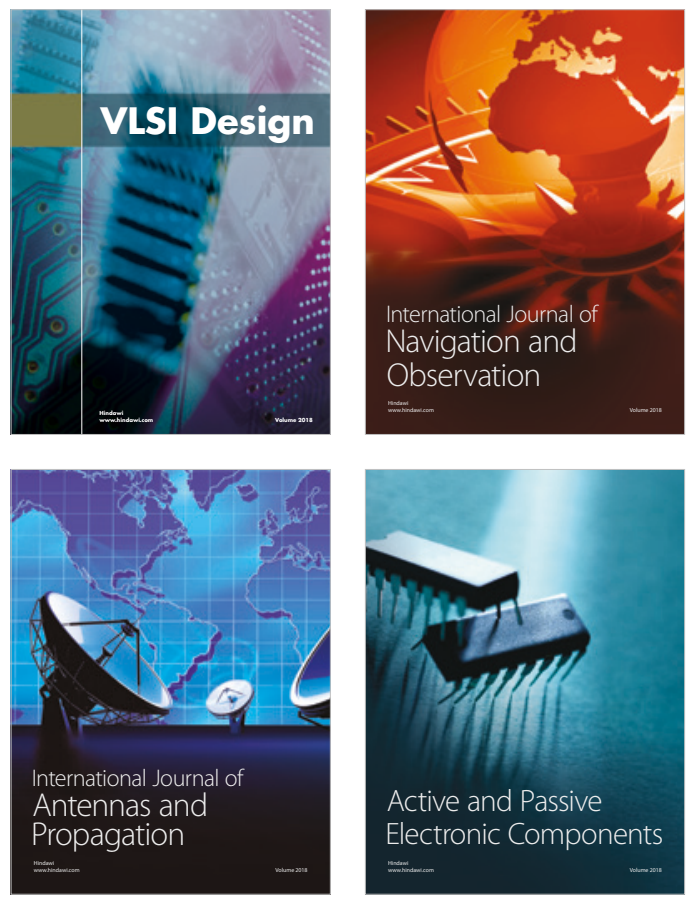
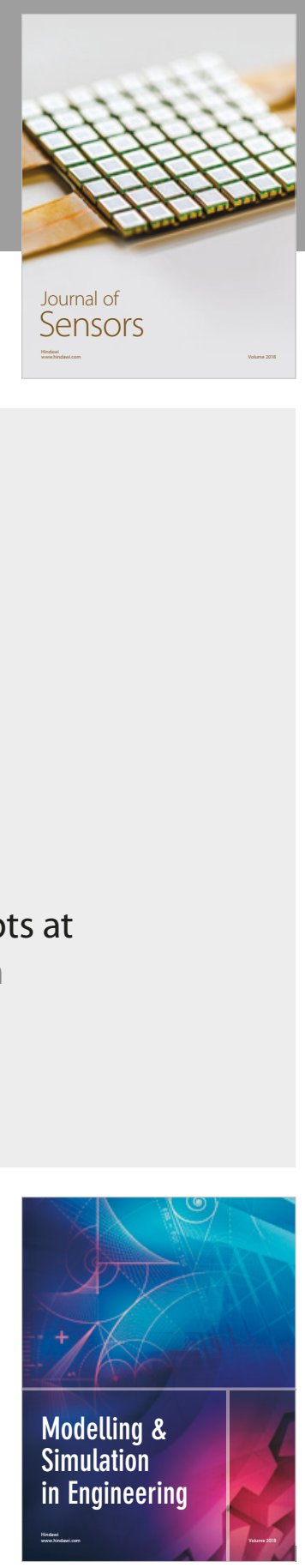

\section{Advances \\ Multimedia}
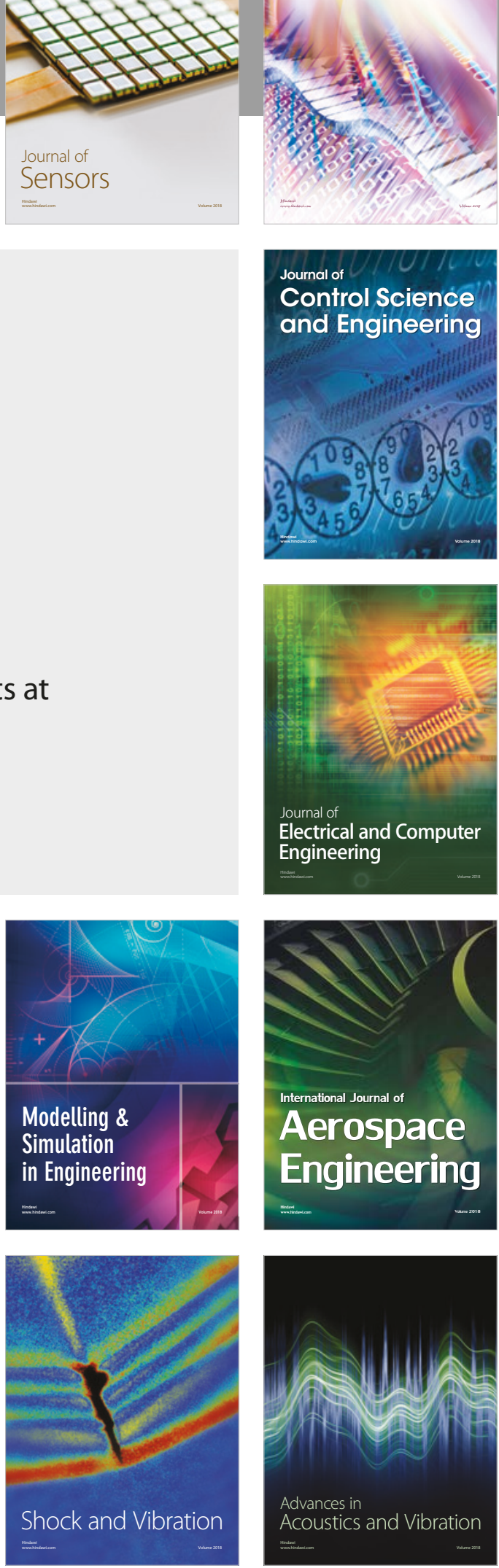